\title{
DEDEKIND DOMAINS AND GRADED RINGS
}

\author{
FABIEN DECRUYENAERE AND ERIC JESPERS
}

(Communicated by Maurice Auslander)

\begin{abstract}
We prove that a Dedekind domain $R$, graded by a nontrivial torsionfree abelian group, is either a twisted group ring $k^{t}[G]$ or a polynomial ring $k[X]$, where $k$ is a field and $G$ is an abelian torsionfree rank one group. It follows that $R$ is a Dedekind domain if and only if $R$ is a principal ideal domain. We also investigate the case when $R$ is graded by an arbitrary nontrivial torsionfree monoid.
\end{abstract}

We fix some notation and terminology. All rings $R$ are commutative with identity 1 , and all semigroups $S$ are torsionfree. In case $S$ is a monoid, we denote by $e$ the identity of $S$ and by $\mathscr{U}(S)$ the group of invertible elements of $S$. For $s \in S$, denote by $\langle s\rangle$ (respectively $\langle s\rangle^{1}$ ) the subsemigroup (respectively submonoid) of $S$ generated by $S$. For more details on semigroups we refer to [3]. We say that $R$ is $S$-graded if $R=\bigoplus_{s \in S} R_{s}$, a direct sum of additive subgroups, such that $R_{s} R_{t} \subseteq R_{s t}$, for all $s, t \in S$. The set $h(R)=\bigcup_{s \in S} R_{s}$ is the set of all homogeneous elements. If $T$ is a subset of $S$, then we put $R_{[T]}=\bigoplus_{t \in T} R_{t}$. Clearly, if $\operatorname{Supp}(R)=\left\{s \in S \mid R_{s} \neq 0\right\}$, the support of $R$, then $R=R_{[\operatorname{Supp}(R)]}$. Obviously $\operatorname{Supp}(R)$ is a monoid if $R$ is a domain. If, moreover, $S$ is a group and $R_{s} R_{t}=R_{s t}$ for all $s, t \in S$, then $R$ is called strongly $S$-graded. If $I$ is an ideal of $R$, we denote by $(I)_{h}$ the ideal generated by all homogeneous elements of $I$. If $I=(I)_{h}$, then $I$ is called a homogeneous ideal of $R$.

If $S$ is a monoid and $R$ is an $S$-graded integral domain, then $Q^{g}(R)=$ $\left\{r c^{-1} \mid r \in R, 0 \neq c \in R_{s}, s \in S\right\}$, the graded quotient ring of $R$; if moreover, $S$ is cancellative, then $Q^{g}(R)$ is $G$-graded, where $G$ is the quotient group of $S$, and its component of degree $e$ is clearly a field. For further details on graded rings we refer to [6].

In recent years there has been a growing interest in divisibility properties of graded rings. For example, in $[1,7]$ graded rings which are factorial domains are investigated, while in $[1,2]$ graded rings that are Krull domains are studied. In this paper we investigate graded rings which are Dedekind domains.

Received by the editors April 10, 1989.

1980 Mathematics Subject Classification (1985 Revision). Primary 13F05, 16A03.

The first author is a research assistant at the National Fund for Scientific Research, Belgium.

The second author is supported in part by NSERC-grant OGP0036631. 
We begin with two elementary lemmas.

Lemma 1. Let $G$ be a torsionfree abelian group, $R$ a G-graded ring and $S=$ $\operatorname{Supp}(R)$. If $R$ is a Dedekind domain, then $S$ is either a group or a torsionfree cancellative monoid with $\mathscr{U}(S)=\{e\}$.

Proof. Since $R$ is a domain and $1 \in R_{e}, S$ is clearly a submonoid of $G$. Suppose $S \neq \mathscr{U}(S)$. Since $T=S \backslash \mathscr{U}(S)$ is an ideal of $S$, it follows that $R_{[T]}$ is an ideal of $R$; and obviously $R / R_{[T]} \cong R_{\mathscr{U}(S)}$. Hence $R_{[T]}$ is a nonzero prime ideal, and thus a maximal ideal of $R$. Therefore $R_{\mathscr{U}(S)}$ is a field. Since $\mathscr{U}(S)$ is a totally ordered group, it follows, using well-known techniques, that $\mathscr{U}(S)=\{e\}$. This finishes the proof.

Lemma 2. Assume $S$ is a nontrivial torsionfree cancellative monoid and $R$ is an $S$-graded Dedekind domain. If $S=\operatorname{Supp}(R)$, then the quotient group $\langle S\rangle$ of $S$ has torsionfree rank 1 .

Proof. Let $Q=Q^{g}(R)$, the graded quotient ring of $R$. Note that $Q$ is also a Dedekind domain. Since $Q$ is $\langle S\rangle$-graded and because $Q_{e}$ is a field, it is well known that $Q$ is a twisted group ring $Q_{e}^{t}[G]$ of a group $G$ over $Q_{e}$. Moreover, $S=\operatorname{Supp}(R)$ yields $G=\langle S\rangle$. Let $F$ be a maximal free subgroup of $G$, then $G / F$ is a torsion group. Therefore, $Q$ is integral over $Q_{e}^{t}[F]$ and since $\operatorname{dim}(Q)=1$ (note that $S$ is nontrivial) we obtain that $\operatorname{dim}\left(Q_{e}^{t}[F]\right)=1$. Now $Q_{e}^{t}[F]$ is isomorphic with a Laurent polynomial ring over the field $Q_{e}$ in $\operatorname{rank}(F)$ variables. It follows that $\operatorname{rank}(F)=1$. The result follows.

We consider the two cases mentioned in Lemma 1 separately. First we assume $\mathscr{U}(S)=\{e\}$.

Proposition 3. Let $R$ be an $S$-graded ring, where $S=\operatorname{Supp}(R)$ is a nontrivial torsionfree cancellative monoid with $\mathscr{U}(S)=\{e\}$. Then $R$ is a Dedekind domain if and only if $R \cong k[X]$, a polynomial ring over a field $k$.

Proof. Let $T=S \backslash\{e\}$. Then $T$ is a nontrivial ideal of $S$. As in Lemma 1, it follows that $R_{[T]}$ is a maximal ideal of $R$. Hence $R_{e} \cong R / R_{[T]}$ is a field.

We first consider the case that $S=\mathbf{N}$, the nonnegative integers. Put $M=$ $R_{\left[\mathrm{N}_{0}\right]}=\bigoplus_{n>0} R_{n}$, the unique maximal homogeneous ideal of $R$. Therefore, the ideal generated by $R_{1}$ is equal to $M^{n}$ for some $n \geq 1$. But then $n=1$, and hence $R_{m}=R_{1} R_{m-1}=R_{1}^{m}$, for every $m \geq 1$. Consequently $R=R_{0} \oplus$ $\sum_{n \geq 1} R_{1}^{n}$. Let $0 \neq r_{1} \in R_{1}$; then $R r_{1}$ is a nonzero homogeneous ideal and thus $R r_{1}=M$. Hence $R_{1}=R_{0} r_{1}$ and for each $n \geq 1, R_{n}=R_{0} r_{1}^{n}$. So $R=\bigoplus_{n \in \mathbf{N}} R_{0} r_{1}^{n}$, a polynomial ring in $r_{1}$ over $R_{0}$.

We now consider the general case. Let $s \in S, s \neq e$, then $\langle s\rangle^{1} \cong \mathbf{N}$. Because of Lemma 2, $G=\langle S\rangle$ has torsionfree rank one. Let $\operatorname{grp}\{s\}$ be the cyclic subgroup of $G$ generated by $s$, and let $\bar{G}=G / \operatorname{grp}\{s\}$. It follows that $R$ is also a $\bar{G}$-graded ring with identity component $R_{\left[\langle s\rangle^{1}\right]}$. The latter follows from the fact that $\mathscr{U}(S)=\{e\}$. It then follows from [1] that $R_{\left[\langle s\rangle^{\prime}\right]}$ is a Krull domain. 
Now since $\bar{G}$ is a torsion group and because $\mathscr{U}(S)=\{e\}$, we obtain that for every $e \neq t \in S$, there exist $n, m \in \mathbf{N}_{0}$ such that $t^{n}=s^{m}$. Consequently, $R$ is integral over $R_{\left[\langle s\rangle^{1}\right]}$ and, therefore, the latter ring is of dimension 1 . Hence $R_{\left[\langle s\rangle^{1}\right]}$ is a Dedekind domain. It follows from the first case that $R_{s}=R_{0} r_{s}$, $0 \neq r_{s} \in R_{s}$. Therefore, $R=R_{e}^{t}[S]$, a twisted monoid ring. We now prove that $S \cong \mathbf{N}$; this will finish the proof. Since $S$ is torsionfree cancellative and has no nontrivial invertible elements, there exists a linear order $<$ on $S$ such that all elements of $S$ are positive. We assert that $S \backslash\{e\}$ has a minimum element for this order. For if not, then $S$ has an infinite descending chain

$$
s_{1}>s_{2}>s_{3}>\cdots>s_{n}>\cdots>e .
$$

But then one obtains the following infinite strictly ascending chain of ideals in $R$ :

$$
\sum_{s \geq s_{1}} R_{s} \subset \sum_{s \geq s_{2}} R_{s} \subset \cdots \subset \sum_{s \geq s_{n}} R_{s} \subset \cdots ;
$$

a contradiction. Let $s_{1}$ be the minimum element in $S \backslash\{e\}$. So $M=\sum_{s \geq s_{1}} R_{s}$ is the unique maximal homogeneous ideal of $R$ and $R R_{s_{1}}=M$. Consequently, for every $s \in S \backslash\{e\}, R_{s} \subset R R_{s_{1}}$ and thus $s s_{1}^{-1} \in S$. If $s_{1}^{n}<s<s_{1}^{n+1}, n \geq 1$, then $1<s s_{1}^{-n}<s_{1}$, a contradiction since $s s_{1}^{-n} \in S$. Therefore,

$$
e<s_{1}<s_{1}^{2}<\cdots<s_{1}^{n}<\cdots
$$

is a strictly ascending chain of elements of $S$ which cannot be refined in $S$. Suppose there exists $t \in S$ such that $t>s_{1}^{n}$ for all $n \in \mathbf{N}$. Then by an argument as above, such a minimal element $t$ exists. But then $t s_{1}^{-1}<t$ and, for every $n \in \mathbf{N}, t s_{1}^{-1}>s_{1}^{n}$; a contradiction. Hence $S=\left\langle s_{1}\right\rangle^{1} \cong \mathbf{N}$.

Proposition 4. Let $R$ be a G-graded ring, where $G=\operatorname{Supp}(R)$ is a nontrivial torsionfree abelian group. If $R$ is a Dedekind domain, then $R \cong k^{t}[G]$, a twisted group ring over a field $k$, and $G$ has torsionfree rank one.

Proof. It follows from Lemma 2 that $G$ is of rank one. Hence to prove the result, it is sufficient to show that $R_{e}$ is a field, or equivalently that $R$ has no nonzero homogeneous prime ideals. So we assume $R_{e}$ is not a field and derive a contradiction.

Let $P$ be a nonzero homogeneous prime ideal. Then $R / P$ is a field and a $G$ graded ring. Therefore $R / P$ is trivially graded, and thus $P=p+\sum_{g \in G \backslash\{e\}} R_{g}$ where $p$ is a maximal ideal of $R_{e}$. Write $P=P(p)$. Conversely, let $p$ be a nonzero prime ideal of $R_{e}$. Then $R p$ is an ideal of $R$ such that $R p \cap R_{e}=p$. Let $M$ be a homogeneous ideal of $R$ maximal with respect to $M \cap R_{e}=p$. One easily verifies that $M$ is a prime ideal of $R$, and thus by the previous $M=P(p)$. Now fix a nonzero prime ideal $p$ in $R_{e}$. Let $T$ be the ring $R$ localized to the multiplicative set $R_{e} \backslash p$. Then $T$ is also a $G$-graded Dedekind domain, and by the above $T$ has only one nonzero homogeneous prime ideal, namely $P(p)$ localized to $R_{e} \backslash p$. We may assume $T=R$. 
So let $R$ be a $G$-graded Dedekind domain with unique nonzero homogeneous prime ideal $P=P(p)=p+\sum_{g \in G \backslash\{e\}} R_{g}$. It follows that for every $0 \neq x \in P \cap$ $h(R)$ there exists $n(x) \geq 0$ such that $R x=P^{n(x)}$. Since $P=\sum_{x \in P \cap h(R)} R x$, we obtain that $P=R x_{g}$ for some $x_{g} \in R_{g}, g \in G$. Assume $g \neq e$. Then $R_{g}=R_{e} x_{g}$ and thus $R R_{g}=R x_{g}=P$. Therefore, for every $h \in G \backslash\{e\}$, $R_{h}=R_{g} R_{g^{-1} h}$ and $p=R_{g} R_{g^{-1}}$. Consequently, $P^{k} \supseteq p+\sum_{n>0} R_{g^{-n}}$, for all $k \geq 1$; a contradiction as $p \neq 0$. Therefore, $g=e$. In this case it follows that $p=R_{e} x_{e}$ and $R_{g}=R_{g} x_{e}$ for $g \neq e$. We obtain $R R_{g}=R R_{g} R x_{e}$. This yields $R=R x_{e}$ and thus $p=R_{e}$, a contradiction. This finishes the proof.

Corollary 5. Let $R$ be a G-graded ring, where $G$ is a torsionfree abelian group with the ascending chain condition on cyclic subgroups; and assume $|\operatorname{Supp}(R)|>$ 1. Then, $R$ is a Dedekind domain if and only if $R \cong k[X]$ or $R \cong k\left[X, X^{-1}\right]$ for some field $k$.

Proof. Since a torsionfree rank one abelian group with the ascending chain condition on cyclic subgroups is free [4], the result follows from Lemmas 1 and 2 and Propositions 3 and 4.

Remark. Obviously, the condition in Proposition 4 is not sufficient, as, for example, a group algebra over an infinitely generated rank one group is not Noetherian.

Also, $G$ does not need to have the ascending chain condition on cyclic subgroups. The following example is taken from [2]. Let $G$ be an arbitrary torsionfree rank one abelian group and $R=k\left[X_{g} \mid g \in G\right]$, a polynomial ring over the field $k$. Obviously $R$ is a unique factorization domain and is $G$-graded with $\operatorname{deg}\left(X_{g_{1}}^{n_{1}} \cdots X_{g_{r}}^{n_{r}}\right)=g_{1}^{n_{1}} \cdots g_{r}^{n_{r}}$. Clearly $Q=Q^{g}(R)=Q_{e}^{t}[G]$ is also a unique factorization domain. Moreover, $Q \cong Q_{e}[\mathbf{Z}]^{t}[G / \mathbf{Z}]$ is integral over $Q_{e}[\mathbf{Z}]$; so all nonzero prime ideals of $Q$ are maximal. Therefore, $Q$ is a Dedekind domain.

We obtain the following generalization of a result of Gilmer [5, Theorem 13.8].

Corollary 6. Let $S$ be a torsionfree cancellative monoid. Assume $R$ is an $S$ graded ring with $|\operatorname{Supp}(R)|>1$. The following conditions are equivalent.

1. $R$ is a Dedekind domain.

2. $R$ is principal ideal domain.

In these cases, $R \cong k^{t}[G]$, where $G$ is a torsionfree rank one group, or $R \cong k[X]$ with $k$ a field.

Proof. We only need to observe that, when $R \cong k^{t}[G] \cong k[\mathbf{Z}]^{t}[G / \mathbf{Z}]$ ( $G$ of rank 1$)$ and $R$ is a Dedekind domain, then $R$ is a principal ideal domain. For this, let $P$ be a prime ideal of $R$. Then $P=R r_{1}+\cdots+R r_{n}$ for some $r_{i} \in R$. Clearly there exists a finitely generated subgroup $H$ of $G$ such that $r_{i} \in R_{[H]}$, for all $1 \leq i \leq n$. Since $G$ is torsionfree of rank one, it follows that $H \cong \mathbf{Z}$, $R_{[H]} \cong k\left[X, X^{-1}\right]$ and therefore $P \cap R_{[H]}=R_{[H]} r$ for some $r \in R_{[H]}$. Hence $P=R r$. The result follows. 
We now consider rings graded by noncancellative monoids.

Proposition 7. Let $R$ be an $S$-graded ring, where $S=\operatorname{Supp}(R)$ is a nontrivial torsionfree noncancellative monoid. If $R$ is a Dedekind domain, then $|\operatorname{Supp}(R)|=2$ and $R=k+M$, where $k$ is a field and $M$ is a maximal ideal of $R$.

Proof. Since $S$ is torsionfree, $S=\bigcup_{\alpha \in \Gamma} S_{\alpha}$, the disjoint union of its cancellative Archimedean subsemigroups, with $\Gamma$ a semilattice. By $\leq$ we denote the partial order relation on $\Gamma$, that is $\alpha \leq \beta$ if $\alpha \beta=\alpha$. As $S$ is noncancellative, $|\Gamma|>1$ and since $S$ is a monoid, $\Gamma$ has a maximum element, say $\delta$, with $e \in S_{\delta}$. Moreover, $R=\bigoplus_{\alpha \in \Gamma} R_{\alpha}$, a semilattice graded ring, where for every $\alpha \in \Gamma, R_{\alpha}=\bigoplus_{s \in S_{\alpha}} R_{s}$. Put $R^{\prime}=\bigoplus_{\alpha \neq \delta} R_{\alpha}$, then $R^{\prime}$ is a nonzero prime, and thus a maximal ideal of $R$. Hence $R_{\delta}$ is a field.

Let $\alpha \neq \delta$ and $P_{\alpha}=\bigoplus_{\beta} R_{\beta}$, where the sum runs over all $\beta$ that are either incomparable with $\alpha$ or $\beta<\alpha$. Then $P_{\alpha}$ is an ideal of $R$ and $R / P_{\alpha}=$ $\bigoplus_{\beta \geq \alpha} R_{\beta} \neq 0$ is a domain. So $P_{\alpha}$ is a maximal ideal in $R$ or $P_{\alpha}=\{0\}$. But in the first case $\bigoplus_{\beta \geq \alpha} R_{\beta}$ would be a field, which is impossible as each element of $R_{\alpha} \subseteq R^{\prime}$ is not invertible. Hence for every $\alpha \neq \delta, P_{\alpha}=\{0\}$, that is $\{\beta \in \Gamma \mid \beta \leq \alpha$ or $\beta$ incomparable with $\alpha\}=\varnothing$. Therefore $\Gamma=\{\delta, \alpha\}$, $\alpha \neq \delta$ and $\alpha \delta=\alpha ; R=R_{\delta} \oplus R_{\alpha}$ and $S=S_{\delta} \cup S_{\alpha}$. Now if $S_{\delta} \neq\{e\}$, then $S_{\delta}$ is a nontrivial torsionfree abelian group and $R_{\delta}$ is a field graded by $S_{\delta}$, which is impossible. So $S=\{e\} \cup S_{\alpha}$. Now since $S$ is not cancellative there exist $s \in S_{\alpha}$ and $t, t^{\prime} \in S$ such that $s t=s t^{\prime}$ and $t \neq t^{\prime}$. But as $S_{\alpha}$ itself is cancellative we obtain that, say, $t=e$ and thus $t^{\prime} \in S_{\alpha}$. This yields that $t^{\prime}$ is an idempotent, and consequently, $S_{\alpha}=G$ is a group.

Let $1_{G}$ be the identity of $G$. We claim that $G=\left\{1_{G}\right\}$. For if not, then $R=\left(R_{e}+R_{1_{G}}\right) \oplus \sum_{g \in G \backslash\{e\}} R_{g}$ is a Dedekind domain graded by the nontrivial torsionfree group $G$ (the identity component being $R_{e}+R_{1_{G}}$ ). Lemma $4 \mathrm{im}$ plies that $R_{e}+R_{1_{G}}$ is a field. A contradiction as $R_{1_{G}}$ is a nonzero ideal of the latter ring. This proves the claim; and therefore $R=R_{e}+R_{1_{G}}$. This finishes the proof.

Note that there are plenty of Dedekind domains of the type $R=k+M$. For example polynomial rings $k[X]$ or power series $k \llbracket X \rrbracket$ where $k$ is a field, or $\mathbf{R}[X, Y] /\left(X^{2}+Y^{2}-1\right)$. In case $R$ is a principal ideal domain we can prove that $R$ is embedded in a formal power series ring and contains a polynomial ring.

Corollary 8. With the notations and assumptions as in Proposition 7. If, moreover, $R$ is a principal ideal domain and $M=R X, X \in R$, then

$$
k[X] \subseteq R=k+M \subseteq k \llbracket X \rrbracket .
$$

Proof. Let $R_{e}=k$, a field, then $R=k+R X$. It follows that $R=k+k X+$ $k X^{2}+\cdots+k X^{n}+X^{n} R X$. Thus for every $r \in R$, and $n \geq 0$, there exist 
$r_{0}, r_{1}, \ldots, r_{n} \in k$ and $b_{n} \in R X$ such that $r=r_{0}+r_{1} X+\cdots+r_{n} X^{n}+b_{n} X^{n}$. One easily verifies that the $r_{i}$ 's are uniquely determined by $r$. Hence we obtain a well-defined map

$$
\varphi: R \rightarrow k \llbracket X \rrbracket: r \mapsto r_{0}+r_{1} X+\cdots+r_{n} X^{n}+\cdots
$$

It follows that $\varphi$ is a ring homomorphism. Moreover, since $\bigcap_{n \in \mathbf{N}} R X^{n}=\{0\}$, $\varphi$ is a monomorphism. This proves the result.

Of course not every principal ideal domain $R$ is of the form $k+M$. Let $R=\mathbf{R}[X]_{\left(X^{2}+X+1\right)}$, that is the localization of $\mathbf{R}[X]$ with respect to the prime ideal generated by $X^{2}+X+1$. Clearly $R$ is a principal ideal domain, and it is easily verified that $R$ is not of the form $k+M$ for some field $k$ and nonzero ideal $M$.

\section{REFERENCES}

1. D. D. Anderson and D. F. Anderson, Divisibility properties of graded domains, Canad. J. Math. 24 (1982), 196-215.

2. D. F. Anderson, Graded Krull domains, Comm. Algebra 7 (1979), 79-106.

3. A. J. Clifford and G. B. Preston, The algebraic theory of semigroups, vol. I, Math. Surv. Mono. vol. 7, Amer. Math. Soc., Providence, RI, 1961.

4. L. Fuchs,Infinite abelian groups, vol. II, Academic Press, New York, 1970.

5. R. Gilmer, Commutative semigroup rings, The University of Chicago Press, Chicago and London, 1984.

6. C. Nastasescu and F. Van Oystaeyen, Graded ring theory, North-Holland, Amsterdam, 1982.

7. P. Wauters, Factorial domains and graded rings, Comm. Algebra (to appear).

Department of Mathematics, K. U. Leuven, 3030 Leuven, Belgium

Department of Mathematics, Memorial University of Newfoundland, St. John's, AlC 5S7 CANADA 